\title{
ON THE ITERATIVE SOLUTION OF LINEAR OPERATOR EQUATIONS WITH SELF-ADJOINT OPERATORS
}

\author{
J. J. KOLIHA \\ (Received 15 October 1968; revised 18 March 1970) \\ Communicated by J. B. Miller
}

\section{Introduction}

In this paper we deal with a linear equation $A u=f$ in a Hilbert space using a general iterative method with a constant iterative operator for the approximate solution. The method has been studied in many papers $[1,2,4,9,13,14]$ and thoroughly treated by Householder [3] for matrix equations and by Petryshyn [7] for operator equations in considerably general and unified manner.

Our aim is to extend and modify Reich's criterion of convergence for selfadjoint non-singular operators (Theorem 1) and derive Theorem 2 concerning self-adjoint singular operators. These theorems enable us to derive sufficient conditions of convergence for a number of particular methods $[1,5,13,14,17]$. Theorems 3 and 4 are generalizations of previous theorems for $K$-positive definite operators and imply some results of $[6,8,10,12]$. Methods I and II include some standard iterative methods as special cases and give a possibility to accelerate the convergence in comparison with these methods. (Some results on acceleration of Gauss-Seidel and successive overrelaxation methods are being prepared for publishing by the author.)

\section{Preliminaries}

$X$ denotes a Hilbert space over the field of real or complex numbers, $[X]$ the space of all continuous linear operators mapping the whole of $X$ into itself. $R(A)$ denotes the range of $A \in[X], N(A)$ the null space of $A$. Let $Y$ be a closed subspace of $X$. For every $A \in[X]$ we define

$$
g l b_{Y}(A)=\inf _{u \neq 0} \frac{\|A u\|}{\|u\|}, \quad l u b_{Y}(A)=\sup _{\substack{u \neq 0 \\ 0}} \frac{\|A u\|}{\|u\|}, \quad u \in Y .
$$

Obviously, $\|A\|=l u b_{X}(A)$. (The subscript $X$ will sometimes be omitted.) The restriction $A_{0}$ of $A$ to $Y$ has a bounded inverse $A_{0}^{-1}$ if and only if $g l b_{Y}(A)>0$ [16]. The spectral radius $r\left(A_{0}\right)$ of the restriction $A_{0}$ is defined as the limit 


$$
r\left(A_{0}\right)=\lim _{n \rightarrow \infty}\left[l u b_{Y}\left(A^{n}\right)\right]^{1 / n} .
$$

If $Y=X$ the definition becomes the usual one, namely, $r(A)=\lim _{n}\left\|A^{n}\right\|^{1 / n}[16]$. If $A \in[X]$ is symmetric on $Y$, i.e. if

we define

$$
(A u, v)=(u, A v) \text { for all } u, v \in Y,
$$

$$
m_{Y}(A)=\inf _{u \neq 0} \frac{(A u, u)}{(u, u)}, \quad M_{Y}(A)=\sup _{u \neq 0} \frac{(A u, u)}{(u, u)}, \quad u \in Y .
$$

If $A \in[X]$ is self-adjoint, i.e. symmetric on $X$, then $\|A\|=\operatorname{Max}(|m(A)|,|M(A)|)$ by $[16]$.

An operator $A \in[X]$ is non-negative on $Y$ if it is symmetric on $Y$ and $m_{Y}(A) \geqq 0$. If, in addition, $(A u, u)=0$ only for $u=0$ on $Y, A$ is positive on $Y$. $A$ is positive definite on $Y$ if it is symmetric on $Y$ and $m_{Y}(A)>0$.

Lemma $1 .{ }^{1}$ If $A \in[X]$ is non-negative on $X$, then

$$
\|A u\|^{2} \leqq\|A\|(A u, u) \text { for all } u \in X .
$$

Proof. Put $\phi(u, v)=(A u, v)$. Since $A$ is self-adjoint and non-negative, $\phi$ is a non-inegative Hermitian form and therefore satisfies the Schwarz inequatity

setting $v=A u$, we get

$$
(\phi(u, v))^{2} \leqq \phi(u, u) \phi(v, v)
$$

$$
(A u, A u)^{2} \leqq(A u, u)\left(A^{2} u, A u\right) \leqq\|A\|(A u, u)(A u, A u),
$$

and

$$
(A u, A u) \leqq\|A\|(A u, u) .
$$

Lemma 2. Let $A \in[X]$ be non-negative on $X$, and let $Y$ be a closed subspace of $X$. Then the restriction $A_{0}$ of $A$ to $Y$ has the inverse $A_{0}^{-1}$ if and only if $A_{0}$ is positive on $Y ; A_{0}^{-1}$ is bounded if and only if $A_{0}$ is positive definite on $Y$.

Proof. For every non-zero $u \in Y$, we have

$$
\|A u\|^{2} \leqq\|A\|(A u, u) \leqq\|A\|\|A u\|\|u\|
$$

by Lemma 1 and the Schwarz inequality. Hence $(A u, u)=0$ if and only if $A u=0$, and $A_{0}^{-1}$ exists if and only if $A$ is positive on $Y$. From the last inequality it follc.ws that

$$
g l b_{Y}^{2}(A) \leqq\|A\| m_{Y}(A) \leqq\|A\| g l b_{Y}(A),
$$

so $g l b_{Y}(A)>0$ if and only if $m_{Y}(A)>0$.

1 Due to T. Reid. 
LemMA 3. Let the restriction $B_{0}$ of $B \in[X]$ to a closed subspace $Y$ of $X$ have a bounded inverse $B_{0}^{-1}$. Let $W \in[X]$ be positive definite on the subspace $Z=B Y$ (the image of $Y$ by $B$ ). Then the operator $B^{*} W B$ is positive definite on $Y$.

Proof. $B^{*} W B$ is symmetric on $Y$ and for every non-zero $u \in Y$,

$$
\frac{\left(B^{*} W B u, u\right)}{(u, u)}=\frac{(W B u, B u)}{(B u, B u)} \cdot \frac{\|B u\|^{2}}{\|u\|^{2}} \geqq m_{Z}(W) \cdot g l b_{Y}^{2}(B)>0 .
$$

This proves the assertion.

Lemma 4. Let $A \in[X]$ be positive definite on a closed subspace $Y$ of $X$ and let $B \in[X]$ be symmetric on $Y$. If $\sigma$ is a real number such that

$$
|\sigma| \operatorname{lu} b_{Y}(B)<m_{Y}(A),
$$

the operator $A+\sigma B$ is positive definite on $Y$.

Proof. For every non-zero $u \in Y$, we have

$\frac{([A+\sigma B] u, u)}{(u, u)}=\frac{(A u, u)}{(u, u)}+\frac{(\sigma B u, u)}{\|u\|^{2}} \geqq m_{\mathrm{Y}}(A)-|\sigma| \frac{\|B u\|}{\|u\|} \geqq m_{Y}(A)-|\sigma| \cdot l u b_{\mathrm{Y}}(B)$

by the Schwarz inequality. Since $A+\sigma B$ is symmetric on $Y$, it is also positive definite on $Y$ if $m_{Y}(A)-|\sigma| l u b_{Y}(A)>0$.

\section{Convergence}

We consider the equation

$$
A u=f,
$$

where $A \in[X]$ is self-adjoint and $f$ is a given vector in $X$. Let $P$ and $P^{-1} \in[X]$. Then the equation

$$
u=(I-P A) u+P f
$$

is equivalent to (1) and we form successive approximations

$$
u_{n+1}=(I-P A) u_{n}+P f, \quad n=0,1,2, \cdots,
$$

starting with an initial approximation $u_{0} \in X$. The process (2) is said to be totally convergent for a fixed $f \in X$ if the sequence $\left(u_{n}\right)$ converges for every $u_{0} \in X$ (not necessarily to the same limit).

It is known [16] that the series

$$
(I-T)^{-1}=\sum_{n=0}^{\infty} T^{n} \in[X]
$$

converges uniformly for $T \in[X]$ with $r(T)<1$. Therefore (2) converges totally to a unique solution $u^{*}$ of (1) for any $f \in X$ if $r(1-P A)<1$, and 


$$
u^{*}=\sum_{n=0}^{\infty}(I-P A)^{n} P f
$$

The following lemma generalizes the result which has been proved by Stein for finite matrices (cf. [3], p. 103). Petryshyn ([11j, Lemma 3) derived similar necessary and sufficient conditions in the case that $T$ is a self-adjoint operator of the form $T=I-\beta K$.

LEMma 5. For $T \in[X], r(T)<1$ if and only if there is a self-adjoint operator $A \in[X]$, positive definite on $X$, such that $A-T^{*} A T$ is also positive definite on $X$.

Lemma 5 is an immediate consequence of the following more general assertion.

Lemma 6. Let $T \in[X]$, let $Y$ be a closed subspace of $X$ invariant under $T$ (i.e. $T Y \subset Y$ ) and let $T_{0}$ be the restriction of $T$ to $Y$. Then $r\left(T_{0}\right)<1$ on $Y$ if and only if there is an operator $A \in[X]$, positive definite on $Y$, such that $A-T^{*} A T$ is also positive definite on $Y$.

Proof. Let $A$ and $H=A-T^{*} A T$ be positive definite on $Y$. Then it may be deduced from Lemma 4 that there is a real number $\sigma, 0<\sigma<1$, such that $H-\sigma A$ is positive definite on $Y$. Then we get

$$
\left(T^{*} A T u, u\right)=([A-H] u, u) \leqq(1-\sigma)(A u, u) \text { for all } u \in Y .
$$

Put $|u|=(A u, u)^{\frac{1}{2}}$ for every $u \in Y$. The norm $|\cdot|$ on $Y$ is equivalent to the restriction of $\|\cdot\|$ to $Y$. Also the associated operator norm $|\cdot|$ on $[Y]$ is equivalent to the restriction of the operator norm $\|\cdot\|$ to $[Y]$. Therefore, since $T \in[Y]$ by assumption,

$$
r\left(T_{0}\right)=\lim _{n \rightarrow \infty}\left\|T_{0}^{n}\right\|^{1 / n}=\lim _{n \rightarrow \infty}\left|T_{0}^{n}\right|^{1 / n} \quad \text { on } Y .^{2}
$$

(4) implies that for every $u \in Y$,

$$
|T u|=(A T u, T u)^{\frac{1}{2}}=\left(T^{*} A T u, u\right)^{\frac{1}{2}} \leqq(1-\sigma)^{\frac{1}{1}}|u|,
$$

and accordingly, $\left|T_{0}\right| \leqq(-\sigma)^{\frac{1}{2}}<1$. Thus $r\left(T_{0}\right) \leqq\left|T_{0}\right|<1$.

Suppose now that $r\left(T_{0}\right)<1$. The number series $\sum_{n=0}^{\infty}\left\|T_{0}^{* n} T_{0}^{n}\right\|$ converges since it satisfies the Cauchy root test:

$$
\lim _{n \rightarrow \infty}\left\|T_{0}^{* n} T_{0}^{n}\right\|^{1 / n} \leqq \lim _{n \rightarrow \infty}\left(\left\|T_{0}^{* n}\right\| \cdot\left\|T_{0}^{n}\right\|\right)^{1 / n}=\lim _{n \rightarrow \infty}\left\|T_{0}^{n}\right\|^{2 / n}=r^{2}\left(T_{0}\right)<1 ;
$$

we have used the relations $T_{0}^{* n}=T_{0}^{n *}$ and $\left\|T_{0}^{*}\right\|=\left\|T_{0}\right\|$ for $T_{0} \in[Y]$ ([16], pp. 249, 250). Therefore the operator series $\sum_{n=0}^{\infty} T_{0}^{* n} T_{0}^{n}$ converges in the sense of the uniform operator topology on $Y$ to a positive definite $A_{0} \in[Y] . A_{0}$ can be extended to an operator $A \in[X] . A-T^{*} A T$ is positive definite on $Y$ as its restriction to $Y$ is the identity operator on $Y$.

Lemma 5 follows from Lemma 6 by putting $Y=X$.

2 Writing $\left\|T_{0}\right\|$ instead of $l u b_{Y}(T)$. 
The following two theorems represent the main objective of the present paper. Theorem 1, dealing with a non-singular oparator $A$. is a generalization of Reich's result for finite matrices (cf. [3], p. 111). Theorem 2 is concerned with the case of a singular $A$.

Theorem 1. Let $A \in[X]$ be self-adjoint, $P$ and $P^{-1} \in[X]$, and let $W=P^{-1}+$ $P^{*-1}-A$ be positive definite on $X$. Then $r(I-P A)<1$ if and only if $A$ is positive definite on $X$.

Proof is given after the proof of

TheOREM 2. Let $A \in[X]$ be self-adjoint, $P$ and $P^{-1} \in[X]$. Let $Y$ be the image of $N(A)^{\perp}$ by $P, T=I-P A$, and let $A_{0}, T_{0}$ be the restrictions of $A$ and $T$ to $Y$ respectively. Let $W=P^{-1}+P^{*-1}-A$ be positive definite on $Y$. Then

(i) $Y$ is invariant under $T$, so that $T_{0} \in[Y]$.

(ii) $Y \cap N(A)=\{0\}$, i.e. $A_{0}^{-1}$ exists (not necessarily bounded).

(iii) $r\left(T_{0}\right)<1$ on $Y$ if and only if $A$ is positive definite on $Y$.

Let, in addition, $A$ be positive definite on $Y$. Then

(iv) $X$ is the direct sum $X=N(A) \oplus Y$ (not necessarily orthogonal). The projectors associated with the sum are the operators

$$
U=\lim _{n \rightarrow \infty} T^{n}, \quad V=\sum_{n=0}^{\infty} T^{n}(I-T)
$$

respectively. (The convergence is understood in the norm topology of $[X]$.)

(v) $R(A)=N(A)^{\perp}$, i.e. (1) is solvable if and only if $f \in N(A)^{\perp}$.

(vi) The iterative process (2) converges tctally if and only if (1) is solvable; the limit $\bar{u}=\lim _{n} u_{n}$ is a solution of (1), and

$$
\bar{u}=U u_{0}+u^{*}
$$

where

$$
u^{*}=\left(P A_{0}\right)^{-1} P f=\sum_{n=0}^{\infty} T^{n} P f .
$$

(vii) Let one of the following conditions be satisfied:

$$
\text { (a) } P A=A P, \quad \text { (b) } P A=A P^{*} \text {. }
$$

Then $Y=N(A)^{\perp}, U$ and $V$ are orthogonal projectors, and $u^{*}$ in (3) is the solution of (1) with the smallest norm for a given $f \in N(A)^{\perp}$.

Proof. (i) Since $A$ is self-adjoint, $\overline{R(A)}=N(A)^{\perp}$, and thus $R(P A) \subset Y$. If $u \in Y$, then $T u=u-P A u \in Y$.

(ii) If $u \in N(A) \cap Y$, then $P^{-1} u \in N(A)^{\perp}$ and 


$$
(W u, u)=\left(P^{-1} u, u\right)+\left(u, P^{-1} u\right)-(A u, u)=0 .
$$

Since $W$ is positive definite on $Y, u=0$.

(iii) By direct computation we obtain

$$
A-T^{*} A T=(P A)^{*} W(P A) .
$$

If $A$ is positive definite on $Y$, then $A_{0}^{-1}$ is bounded, since $g l b_{Y}(A) \geqq m_{Y}(A)$. $\left(P A_{0}\right)^{-1}$ is also a bounded operator, and $H=(P A)^{*} W(P A)$ is positive definite on $Y$ by Lemma 3. The direct part of Lemma 6 gives $r\left(T_{0}\right)<1$ on $Y$.

Suppose now $r\left(T_{0}\right)<1$ on $Y$. Then the restriction $P A_{0}$ of $P A$ to $Y$ has a bounded inverse

$$
\left(P A_{0}\right)^{-1}=\sum_{n=0}^{\infty} T_{0}^{n} \in[Y] .
$$

Thus, according to Lemma $3, H=(P A)^{*} W(P A)$ is positive definite on $Y$. The proof of the assertion (iii) will be accomplished when we show that

$$
(A u, u)=(H u, u)+\sum_{k=1}^{\infty}\left(H T^{k} u, T^{k} u\right), \quad u \in Y .
$$

Since $A=H+T^{*} A T$ by (6), we can verify by induction that

and

$$
A=\sum_{k=0}^{n} T^{* k} H T^{k}+T^{* n+1} A T^{n+1}
$$

$$
(A u, u)=\sum_{k=0}^{n}\left(H T^{k} u, T^{k} u\right)+\left(A T^{n+1} u, T^{n+1} u\right)
$$

for every $u \in X$. If $u \in Y,\left(A T^{n+1} u, T^{n+1} u\right) \rightarrow 0$ and (8) is true.

(iv) Since $A$ is positive definite on $Y$ by assumption, $r\left(T_{0}\right)<1$ on $Y$ by (iii). Thus $\left(P A_{0}\right)^{-1} \in[Y]$ by $(7)$ and the composite operator $V=\left(P A_{0}\right)^{-1} P A$ is well defined as $R(P A) \subset Y$. Obviously,

$$
\left(P A_{0}\right)^{-1} P A=\sum_{n=0}^{\infty} T^{n}(I-T) \in[X, Y] \subset[X],
$$

where $[X, Y]$ denotes the space of all linear continuous operators from $X$ into $Y$. $V$ is a projector by the definition $([16]$, p. 241$)$ if $V \in[X]$ and $V^{2}=V$. Indeed,

$$
V^{2}=\left(P A_{0}\right)^{-1} P A\left(P A_{0}\right)^{-1} P A=\left(P A_{0}\right)^{-1} P A=V,
$$

since $P A\left(P A_{0}\right)^{-1}$ is the identity operator on $Y$. Further,

$$
\begin{aligned}
& R(V)=R\left(\left(P A_{0}\right)^{-1} P A\right)=Y, \\
& N(V)=N\left(\left(P A_{0}\right)^{-1} P A\right)=N(A),
\end{aligned}
$$

hence $V$ is a projector of $X$ onto $Y$ and 


$$
X=N(A) \oplus Y
$$

([16], p. 241). The identity

$$
T^{n}=I-\sum_{k=0}^{n-1} T^{k}(I-T)
$$

implies that $U=\lim _{n} T^{n}=I-V$ and thus $U$ is the complementary projector of $V$ which projects $X$ onto $N(A)$.

(v) As $\overline{R(A)}=N(A)^{\perp}$, we have only to prove that $R(A)$ is closed. In virtue of (9), $R(A)=R\left(A_{0}\right)$, and $A_{0}^{-1}$ maps $R(A)$ continuously onto $Y$; since $Y$ is closed, so is $R(A)$ by the well known property of a continuous mapping.

(vi) From (2) with $T=I-P A$ we derive

$$
u_{n}=T^{n} u_{0}+\sum_{k=0}^{n-1} T^{k} P f
$$

according to (iv) $T^{n} u_{0} \rightarrow U u_{0}$ for every $u_{0} \in X$, and hence $\left(u_{n}\right)$ converges if and only if the series

$$
\sum_{k=0}^{\infty} T^{k} P f
$$

converges. If $f \in N(A)^{\perp}$, the series (11) converges to the sum $\left(P A_{0}\right)^{-1} P f$. On the other hand, if (11) converges, $T^{k} P f \rightarrow U P f=0$ and $P f \in N(U)=R(V)=Y$, i.e. $f \in N(A)^{\perp}$. (5) then follows from (10).

(vii) (a) Let $P A=A P$. Then, in view of (v), $Y=R(P A)=R(A P)=R(A)=$ $N(A)^{\perp}$. Let us remark that since necessarily $U^{*}=U, T^{* n}-T^{n} \rightarrow 0$ in weak operator topology on $X$.

(b) Let $P A=A P^{*}$. Then $T^{*}=T$ and also $U^{*}=U$. Hence $U$ is an orthogonal projector and so is $V$; thus $Y=N(A)^{\perp}$.

Let either $P A=A P$ or $P A=A P^{*}$. Then the sum $u^{*}$ of (11) lies in $N(A)^{\perp}$ for every $f \in N(A)^{\perp}$. Every solution $\bar{u}$ of (1) is of the form (5) with some $u_{0} \in X$, and

$$
\|\bar{u}\|^{2}=\left\|U u_{0}\right\|^{2}+\left\|u^{*}\right\|^{2} \geqq\left\|u^{*}\right\|^{2}
$$

since $U u_{0} \in N(A)$ and $u^{*} \in N(A)^{\perp}$.

Proof of Theorem 1. If $A$ is positive definite on $X, N(A)=\{0\}, Y=X$, and the conclusion about $r(I-P A)$ follows from (iii).

On the other hand, if $r(I-P A)<1$, the operator $P A$ has a bounded inverse $(P A)^{-1}$. Since $P^{-1}$ exists, so does $A^{-1}, N(A)=\{0\}, Y=X$, and (iii) applies.

Remark 1. (a) For $A$ in the form $A=D+S+Q$ with $D$ self-adjoint and $P=(D+\omega S)^{-1} \in[X]$ (for a real number $\omega$ ), Theorem 1 has been proved by Petryshyn [8] for a more general class of $K$-p.d. operators (for more detailed account see Remark 2 of this paper). 
(b) In paper [11], Petryshyn proved among other things the assertion (iv) of Theorem 2 (concerning the projection operator $U$ ) under the condition that $P$ is of the form $P=\beta I$; he also proved part (v) of Theorem 2 for this special case.

(c) The assertion (iv) of Theorem 2 concerning the projector $U$ has been proved under considerably more general conditions in [2].

(d) For $A$ in the form $A=D-S-Q$ with $D$ self-adjoint and $P=$ $(D-\omega S)^{-1} \in[X](\omega>0)$, Petryshyn [6] has proved part (vi) of Theorem 2 with the assumption (vii) $A P=P A$, using the additional condition that the spectrum $\sigma(T)$ of $T=(D-\omega S)^{-1}[(1-\omega) D+\omega Q]$ contains only eigenvalues of finite multiplicity with zero as its sole possible limit point.

(e) Weissinger has proved Theorem 2 (i), (ii), (v) and (vi) in the Hauptsatz of [17] for the Gauss-Seidel method (see Table 1) under the condition that the operators are either finite matrices or Fredholm integral operators. In his proofs, however, he hardly uses this restriction. The method used in the proof of the direct part of our Lemma 6 is essentially that employed in the proof of Satz 1 in [17]. The identity (6) of this paper is a modification of (2.5) in the quoted paper.

Now we apply our results to an abstract Fredholm equation

$$
u-B u=f,
$$

where $B$ is self-adjoint and completely continuous on $X$. Obviously, (12) is an equation of the type (1) with $A=I-B$. Let $N$ be the null space of $I-B$, i.e. the subspace of all eigenvectors of $B$ corresponding to an eigenvalue 1. It is known that $N$ is of finite dimension. Let $N^{\perp}$ be the orthogonal complement of $N$ and $Y$ the image of $N^{\perp}$ by $P$, where $P$ and $P^{-1} \in[X]$.

Corollary. If $I-B$ is non-negative on $X$ (i.e. $M(B) \leqq 1$ ) and $W=P^{-1}+$ $+P^{*-1}-I+B$ positive definite on $Y$, then the iterative process

$$
u_{n+1}=(I-P+P B) u_{n}+P f, \quad n=0,1, \cdots,
$$

converges totally to a solution $\bar{u}$ of (12) if and only if $f \in N^{\perp}$.

Proof. In view of Theorem 2, we have only to prove that $I-B$ is positive definite on $Y . N \cap Y=\{0\}$ by Theorem 2 (ii), therefore $(I-B)^{-1}$ exists and is bounded as $B$ is completely continuous ([16], p. 281). According to Lemma 2, $I-B$ is positive definite.

Let us remark that if 1 is not an eigenvalue of $B$, then $I-B$ is positive on $X$ and thus, according to Lemma 2 , positive definite on $X$; in this case $r(I-P+P B)<1$.

\section{Applications to $K$-positive definite operators}

Let $K \in[X]$ be a fixed positive definite operator on $X$ throughout this section. An operator $A \in[X]$ is called $K$-symmetric on a closed subspace $Y$ of $X$ if

$$
(A u, K v)=(K u, A v) \text { for all } u, v \in Y,
$$


and $K$-positive definite $(K$-p.d.) on $Y$ if, for some positive $\alpha$,

(cf. $[6,8,10]$ ).

$$
(A u, K u) \geqq \alpha\|u\|^{2} \quad \text { for all } u \in Y
$$

It is known [10] that $A \in[X]$ is $K$-symmetric on $X$ if and only if $A^{*} K=K A$, and $K$-p.d. on $Y$ if $A$ is $K$-symmetric on $Y$ and $K A$ is positive definite on $Y$.

To obtain an analogue of Theorem 1 for $K$-symmetric $A$, we replace $A$ in Theorem 1 by $K A$ and $P$ by $P K^{-1}$. Then $W$ in Theorem 1 bzcomes $K P^{-1}+P^{*-1} K-$ $K A$ while $I-P A$ does not change. Obviously, $K P^{-1}+P^{*-1} K-K A$ is positive definite on $X$ if and only if $P^{-1}+K^{-1} P^{*-1} K-A$ is $K$-p.d. on $X$. Expressing the result of this substitution in terms of $K$-symmetry and $K$-positive definiteness, we obtain the following

Theorem 3. Let $A \in[X]$ be K-symmetric on $X, P$ and $P^{-1} \in[X]$ and let $W=P^{-1}+K^{-1} P^{*-1} K-A$ be $K-p . d$. on $X$. Then $r(I-P A)<1$ if and only if $A$ is $K$-p.d. on $X$.

Similar substitution applied to Theorem 2 gives

Theorem 4. Let $A \in[X]$ be $K$-symmetric on $X, P$ and $P^{-1} \in[X]$. Denote $Y$ the image of $N(A)^{\perp}$ by $P K^{-1}, T=I-P A$, and $A_{0}, T_{0}$ the restrictions of $A$ and $T$ to $Y$, respectively. Let $W=P^{-1}+K^{-1} P^{*-1} K-A$ be $K-p . d$. on $Y$. Then:

(i) As in Theorem 2.

(ii) As in Theorem 2.

(iii) $r\left(T_{0}\right)<1$ on $Y$ if and only if $A$ is $K-p$.d. on $Y$.

Let, in addition, $A$ be $K$-p.d. on $Y$. Then:

(iv) As in Theorem 2.

(v) $R(K A)=N(A)^{\perp}$, i.e. (1) is solvable if and only if $K f \in N(A)^{\perp}$.

(vi) As in Theorem 2.

Proof. If we replace $A$ by $K A, f$ by $K f$ and $P$ by $P K^{-1}$ in Theorem 2, the equation (1) becomes $K A u=K f$ which is equivalent to (1). The iterative process (2) for the new setting coincides with the original one:

$$
\begin{aligned}
& u_{n+1}=\left(I-\left(P K^{-1}\right)(K A)\right) u_{n}+\left(P K^{-1}\right)(K f), \\
& u_{n+1}=(I-P A) u_{n}+P f .
\end{aligned}
$$

Finally, $N(K A)=N(A)$.

\section{Special cases}

The iterative process (2) is fully determined when the operator $P$ is chosen. For every particular method, we find the iterative operator $T=I-P A$ and the auxiliary operator $W$. Then we can apply Theorem 1 and 2 or 3 and 4 . 
We make use of the following modification of a given method. The iterative process (2) is extrapolated if we replace the operator $P$ by $\phi P$ (usually $\phi>0$ ). For this concept and general theorem on convergence of extrapolated processes see [15]. The iterative operator of the extrapolated method is $I-\phi P A$, and under certain conditions $\phi$ may be found that $r(I-\phi P A)$ is less than $r(I-P A)$ and the rate of convergence is increased (cf. $[10,12,15])$.

Method $I$. Let $A \in[X]$ be decomposed in $[X]$ into the form

(Ia) $A=D-B-F, B=L+L^{*}$, where

(Ib) $D$ and $F$ are self-adjoint.

Let further $\alpha$ and $\beta$ be real constants such that

(Ic) $P=(\alpha D+\beta L)^{-1}$ exists on $X$,

(Id) $W=(2 \alpha-1) D+(\beta+1) B+F$ is positive definite on the image $Y$ of $N(A)^{\perp}$ by the operator $P=(\alpha D+\beta L)^{-1}$.

Proposition 1. If the operators $D, L, F$ and the numbers $\alpha, \beta$ satisfy the conditions (la), (Ib), (Ic) and (Id), then the basic conditions of Theorem 2 (and Theorem 1) are satisfied, i.e.

(i) $A$ is self-adjoint,

(ii) $P$ and $P^{-1} \in[X]$,

(iii) $W_{1}=P^{-1}+P^{*-1}-A$ is positive definite on $Y$.

The iterative operator $T$ of Method I is

$$
T=(\alpha D+\beta L)^{-1}\left[(\alpha-1) D+(\beta+1) L+L^{*}+F\right] .
$$

Proof. (i) From (Ia) and (Ib) it follows that $A$ is self-adjoint.

(ii) $P^{-1} \in[X]$ by (Ic). Since the inverse $P=(\alpha D+\beta L)^{-1}$ exists on the whole of $X, P$ is bounded by the Banach theorem.

(iii) One can easily verify that $W_{1}=P^{-1}+P^{*-1}-A=(2 \alpha-1) D+$ $(\beta+1) B+F=W$ with $P$ defined in (Ic). The iterative operator is $T=I-P A=$ $P\left(P^{-1}-A\right)$, and this gives (13) for $P=(\alpha D+\beta L)^{-1}$.

In case that $D=I$ and $r(L)=0$, (Ic) is satisfied for all $\alpha \neq 0$ and $\beta \neq 0$ since then

$$
(\alpha I+\beta L)^{-1}=\sum_{n=0}^{\infty}(-1)^{n} \alpha^{-n-1} \beta^{n} L^{n} \in[X] .
$$

The equality $r(L)=0$ is satisfied if $L$ is a strictly triangular matrix in $n$-dimensional Euclidean space or a Volterra integral operator in $L^{2}[a, b]$. 
Specializing constants $\alpha$ and $\beta$, we obtain Table 1. Methods (i)-(iii) are well known standard methods; the parameters $\omega$ and $\phi$ (overrelaxation and extrapolation factors respectively) are assumed to be positive.

Proposition 2. If the operators $D, L, F$ and the numbers $\alpha, \beta$ satisfy the conditions $(\mathrm{Ia}),(\mathrm{Ib}),(\mathrm{Ic})$, together with

(Ie) $D$ is positive definite on $Y$,

and

(If) $\alpha>\frac{1}{2}, l u b_{Y}((\beta+1) B+F)<(2 \alpha-1) m_{Y}(D)$

or more restrictive condition

(If) $\alpha>\frac{1}{2},|\beta+1| \operatorname{lu} b_{Y}(B)+\operatorname{lu} b_{Y}(F)<(2 \alpha-1) m_{Y}(D)$,

then the auxiliary operator

$$
W=P^{-1}+P^{*-1}-A=(2 \alpha-1) D+(\beta+1) B+F
$$

is positive definite on $Y$.

Proof. Since $\alpha>\frac{1}{2}$, the operator $(2 \alpha-1) D$ is also positive definite. Then we use Lemma 4 with $\sigma=1,(2 \alpha-1) D$ in place of $A$ and $(\beta+1) B+F$ in place of $B$, to infer that $W$ is positive definite if (If) is satisfied. (If) follows from (If)' since $l u b(\lambda M+\kappa N) \leqq|\lambda| l u b(M)+\kappa|| l u b(N)$ for any two $M, N \in[X]$, and any two real $\lambda, \kappa$.

Two special cases of Method I are of a particular interest in numerical applications:

(A) The operators in (Ia) are matrices in $n$-dimensional Euclidean space. $D$ is usually the diagonal of $A, L$ the lower triangle of $A, F=0$. The equation (1) can be transformed (if $A$ is irreducible) into the equivalent equation whose matrix has the unit diagonal entries, i.e. $D=I$.

(B) The equation (1) is a Fredholm equation of the form (12) (hence $D=I$ ), where $B=L+L^{*}$, and $L$ is a Volterra operator ( $L$ is completely continuous on $X$ and $r(L)=0$ by the definition), $F=0$. If (12) is ail integral equation in $L^{2}[a, b]$, then the operators $B$ and $L$ are defined by

$$
(B u)(t)=\int_{a}^{b} b(t, s) u(s) d s, \quad(L u)(t)=\int_{a}^{t} b(t, s) u(s) d s,
$$

where $b(t, s)=\overline{b(s, t)}$ is a kernel from $L^{2}([a, b] \times[a, b])$.

The following Table 2 gives sufficient conditions for positive definiteness of the auxiliary operator $W$ in (15) under the conditions (la), (Ib), (Ic) and (le) with $F=0$. The conditions for the extrapolated Gauss-Seidel and overrelaxation methods are presented for the first time. The table is derived from (If). 


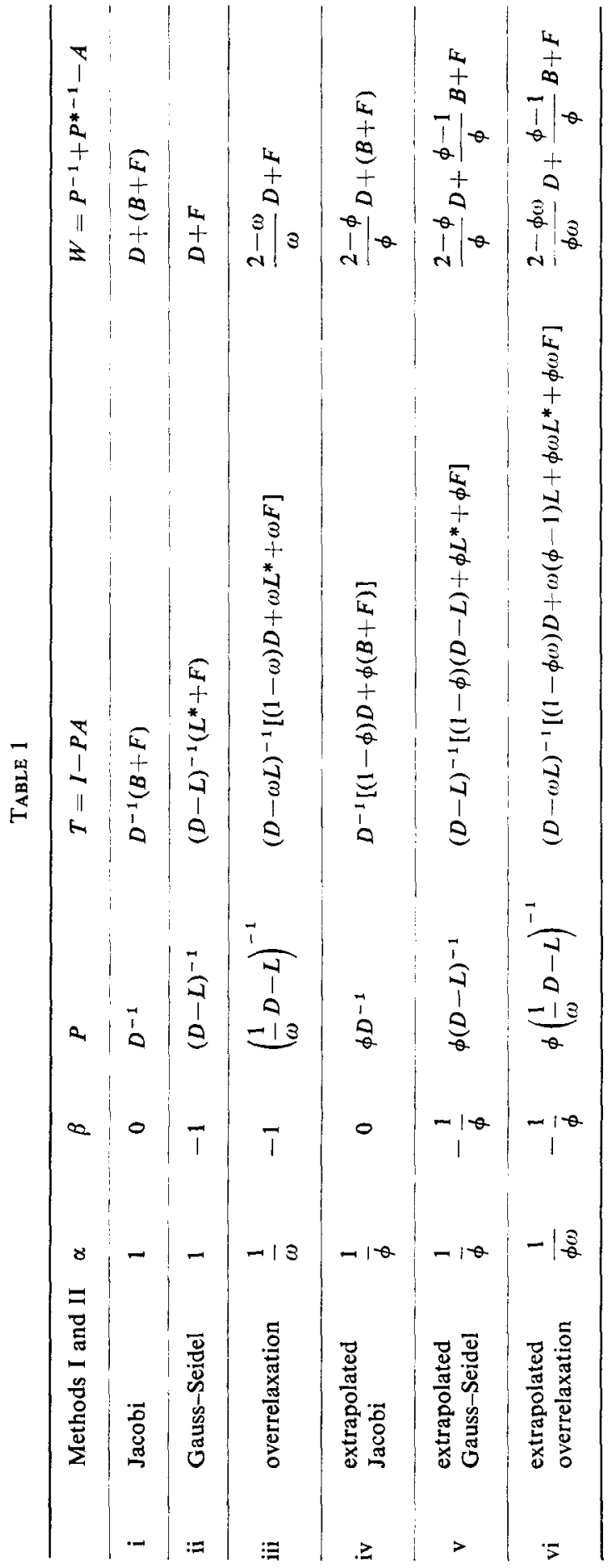


TABLE 2

\begin{tabular}{llll}
\hline i & $\|B\|<m$ & iv & $0<\phi<\frac{2 m}{\|B\|+m}$ \\
\hline ii $\quad$ fulfilled automatically & v & $\frac{(\|B\|-2 m)^{+}}{\|B\|-m}<\phi<\frac{\|B\|+2 m}{\|B\|+m}$ \\
\hline iii $\quad 0<\omega<2$ & vi & $\frac{\left(\|B\|-2 \omega^{-1} m\right)^{+}}{\|B\|-m}<\phi<\frac{\|B\|+2 \omega^{-1} m}{\|B\|+m}$ \\
\hline
\end{tabular}

Notation: a) $m$ stands for $m(D)$.

b) $a^{+}$means $\operatorname{Max}(a, 0)$ for any real $a$.

c) The symbol 0/0, which can appear in the formulae (v) and (vi), is to be taken as 0 .

Method II. Suppose that $K$ is an operator as in Section 4, i.e.

(IIa) $K \in[X]$ is positive definite.

Let $A \in[X]$ be decomposed in $[X]$ into the form

(IIb) $A=D-B-F, B=L+L^{*}$

where

(IIc) $A, D$ and $F$ are $K$-symmetric on $X$,

(IId) $L K=K L$.

Let further $\alpha, \beta$ be real constants such that

(Ile) $P=(\alpha D+\beta L)^{-1}$ exists on $X$,

(IIf) $W=(2 \alpha-1) D+(\beta+1) B+F$ is $K$-p.d. on the image $Y$ of $N(A)^{\perp}$ by $(K(\alpha D+\beta L))^{-1}$.

Proposition 3. If the operators $K, D, L, F$ and the numbers $\alpha, \beta$ satisfy the conditions (IIa), (IIb), (IIc), (IId), (Ile) and (IIf), then the basic conditions of Theorem 4 (and thus Theorem 3) are satisfied, i.e.

(i) $A$ is $K$-symmetric on $X$,

(ii) $P$ and $P^{-1} \in[X]$,

(iii) $W_{1}=P^{-1}+K^{-1} P^{*-1} K-A$ is $K-p$.d. on $Y$.

The iterative operator $T$ is given by (13).

Proof. (i) $A$ is $K$-symmetric as required in (IIc).

(ii) As in the proof of Proposition 1.

(iii) Note that $D^{*} K=K D$ by (IIc) and Section 4 , and that also $L^{*} K=K L^{*}$ in view of (IId). Then 


$$
\begin{aligned}
W_{1}= & P^{-1}+K^{-1} P^{*-1} K-A=(\alpha D+\beta L)+K^{-1}\left(\alpha D^{*}+\beta L^{*}\right) K-A \\
& =(\alpha D+\beta L)+\left(\alpha D+\beta L^{*}\right)+\left(-D+L+L^{*}+F\right) \\
& =(2 \alpha-1) D+(\beta+1) B+F=W .
\end{aligned}
$$

REMARK 2. (a) Method I (ii). If we take $F=0$, then the necessary and sufficient conditions for the convergence established in Theorem 1, were given in [5]. Weissinger [17] has proved an essential part of Theorem 2.

(b) Method $I$ (iii). If $F=0, D=I$ and the operators are finite matrices, the restriction $0<\omega<2$ in Table 2 is a well known condition for the convergence of the overrelaxation method; Ostrowski proved it necessary and sufficient provided that $A$ is positive definite (cf. [3], p. 112).

(c) Method I (iv) is basically identical with that of Wiarda. This method for operators in Hilbert and Banach spaces has been studied by Schönberg [14]. The sufficient conditions given there are formulated in terms of the operator norm.

(d) Method $I I$ (iii) has been investigated by Petryshyn $[6,8,12]$. The necessary and sufficient conditions of the Main Theorem in [8] are essentially the same as those of our Theorem 3 applied to Method II (iii):

THEOREM (Petryshyn). Suppose that $K \in[X]$ is positive definite, and that $A \in[X]$ is decomposed in $[X]$ into the form

$$
A=D+S+Q
$$

where $S$ commutes with $K$ and $D$ is $K$-symmetric. Let $\omega$ be a positive real number such that $(D+\omega S)$ has a bounded inverse on all of $X$ and the operator

$$
G=\frac{2-\omega}{\omega} D+S^{*}-Q
$$

is $K$-p.d. on $X$. Then a necessary and sufficient condition that the spectrum $\sigma(T)$ of the operator

$$
T=(D+\omega S)^{-1}((\omega-1) D+\omega Q)
$$

lies in the interior of the unit circle is that $A$ be K-p.d.

If we put $L=-S, F=S^{*}-Q, P=(1 / \omega) D+S$, then we see that the auxiliary operator $W$ in (15) is identical with $G$ in the Theorem. We can easily verify that the conditions (IIa), (IIb), (IIc), (IId), (IIe) and (IIf) are satisfied.

Methods II (ii), I (ii) and I (iii) are special cases of Method II (iii) ( $\omega=1$, $\omega=1$ together with $K=I$, and $K=I$ respectively), and the necessary and sufficient conditions for their convergence equivalent to those given in our Theorem 3 are also given in [8] and [12].

(e) Method II (iv). Theorem 2 in Petryshyn's paper [10] is a somewhat stronger analogue of our Theorem 3 applied to Method II (iv) in the case that 
the operators are finite matrices. (In Petryshyn's notation $B+F=-Q$.) Theorem 3 of [12] gives the same necessary and sufficient conditions as our Theorem 3 applied to the extrapolated Jacobi method. [12] presents further generalizations of the method.

(f) Method II (v) has been suggested by Sisler [15] for finite matrices. The sufficient conditions for the convergence given in [15] are presented in terms of spectral radii.

\section{References}

[1] H. Bialy, 'Iterative Behandlung linearer Funktionalgleichungen', Archive Rational Mech. Anal. 4 (1959), 166-176.

[2] F. E. Browder and W. V. Petryshyn, 'The solution by iteration of linear functional equations in Banach spaces', Bull. Amer. Math. Soc. 72 (1966), 566-570.

[3] A. S. Householder, The Theory of Matrices in Numerical Analysis (Blaisdell Publ. Co. 1965).

[4] H. B. Keller, 'On some iterative methods for solving elliptic difference equations', Quart. Appl. Math. 16 (1958), 209-226.

[5] S. G. Krein and O.I. Prozorovskaja, 'An analogue of Seidel's method for operator equations', Voronez. Gos. Univ., Trudy Sem. Funkcional. Anal. 5 (1957), 35-38.

[6] W. V. Petryshyn, 'The generalized overrelaxation method for the approximate solution of operator equations in Hilbert space', J. Soc. Indust. Appl. Math. 10 (1962), 675-690.

[7] W. V. Petryshyn, 'Direct and iterative methods for the solution of linear operator equations in Hilbert space', Trans. Amer. Math. Soc. 105 (1962), 136-175.

[8] W. V. Petryshyn, 'On the generalized overrelaxation method for operation equations', Proc. Amer. Math. Soc. 14 (1963), 917-924.

[9] W. V. Petryshyn, 'On a general iterative method for the approximate solution of linear operator equations', Math. Comp. 17 (1963), 1-10.

[10] W. V. Petryshyn, 'On extrapolated Jacobi or simultaneous displacement method in the solution of matrix and operator equations', Math. Comp. 19 (1965), 37-55.

[11] W. V. Petryshyn, 'On generalized inverses and on the uniform convergence of $(I-\beta K)^{n}$ with applications to iterative methods', J. Math. Anal. Appl. 18 (1967), 417-439.

[12] W. V. Petryshyn, 'Remarks on the generalized overrelaxation and the extrapolated Jacobi methods for operator equations in Hilbert space', J. Math. Anal. Appl. 29 (1970), $558-568$.

[13] L. B. Rall, 'Error bounds for iterative solutions of Fredholm integral equation', Pacific $J$. Math. 5 (1955), 977-986.

[14] M. Schönberg, 'Sur la méthode d'itération de Wiarda et Bückner pour la résolution de l'équation de Fredholm I, II', Acad. Roy. Belgique, Bull. Cl. Sci. 37 (1951), 1141-1156, 38 (1952), 154-167.

[15] M. Sisler, 'Úber die Konvergenzbeschleunigung verschiedener Iterationsverfahren', $A p l$. Mat. 12 (1967), 255-266.

[16] A. E. Taylor, Introduction to Functional Analysis (J. Wiley 1958)

[17] J. Weissinger, 'Verallgemeinungen des Seidelschen Iterationsverfahrens', Z. Angew. Math. Mech. 33 (1953), 155-163.

Department of Mathematics

University of Melbourne 